\title{
Disparities in H1N1 Vaccination Rates: a Systematic Review and Evidence Synthesis to Inform COVID-19 Vaccination Efforts
}

\author{
Chelsea K. Ayers, MPH ${ }^{7}$, Karli K. Kondo, $P h D^{1,2}$, Beth E. Williams, MSN, MPH ${ }^{1}$, \\ Devan Kansagara, MD, MCR ${ }^{1,2}$, Shailesh M. Advani, MD, MPH ${ }^{3,4}$, Mia Smith, MPH', \\ Sarah Young, $\mathrm{MPH}^{1}$, and Somnath Saha, $\mathrm{MD}, \mathrm{MPH}^{1,2}$ \\ 'VA Evidence Synthesis Program, VA Portland Health Care System, 3710 SW US Veterans Hospital Rd, Portland, OR, USA; ${ }^{2}$ Oregon Health \& Science \\ University, Portland, OR, USA; ${ }^{3}$ Georgetown University, Washington, DC, USA; ${ }^{4}$ Social Epidemiology Research Unit, Social Behavioral Research \\ Branch, National Human Genome Research Institute, National Institutes of Health, Bethesda, MD, USA; ${ }^{5}$ Oregon Health Authority, Portland, OR, USA.
}

BACKGROUND: Data suggest that there were disparities in $\mathrm{H} 1 \mathrm{~N} 1$ vaccine uptake, and these may inform COVID-19 vaccination efforts. We conducted a systematic review to evaluate disparities in $\mathrm{H} 1 \mathrm{~N} 1$ vaccine uptake, factors contributing to disparities, and interventions to reduce them. METHODS: We searched English-language articles in MEDLINE ALL, PsycINFO, Cochrane Database of Systematic Reviews, and Cochrane Central Register of Controlled Trials from database inception through May 8, 2020. Observational studies examining $\mathrm{H} 1 \mathrm{~N} 1$ vaccine uptake by race/ethnicity, socioeconomic status, rurality, and disability status in US settings were included. Two reviewers independently assessed study eligibility. Single-reviewer data abstraction was confirmed by a second reviewer. We conducted independent dual quality assessment, and collective strength of evidence assessment.

RESULTS: We included 21 studies. African American/ Black, Latino, and low-socioeconomic status participants had disproportionately lower $\mathrm{H} 1 \mathrm{~N} 1$ vaccination rates (low- to moderate-strength evidence). However, Latinos were more likely than Whites to intend to be vaccinated, and African American/Blacks and participants with lower-socioeconomic status were just as likely to intend to be vaccinated as their White and higher-socioeconomic status counterparts (low-strength evidence). Vaccine uptake for other groups has been insufficiently studied. Factors potentially contributing to disparities in vaccine uptake included barriers to vaccine access, inadequate information, and concerns about vaccine safety and efficacy. Studies were largely cross-sectional. Many of the studies are a decade old and were conducted in the context of a different pandemic. The categorization of racial and ethnic groups was not consistent across studies and not all groups were well-studied.

DISCUSSION: Efforts to avoid disparities in COVID-19 vaccination uptake should prioritize vaccine accessibility and convenience in African American/Black, Latino, and low-SES communities; engage trusted stakeholders to

Prior Presentations No prior presentations

Received February 3, 2021

Accepted March 10, 2021

Published online March 31, 2021 share vaccine information; and address concerns about vaccine safety and efficacy.

PRIMARY FUINDING SOURCE: Department of Veterans Affairs, Veterans Health Administration, Health Services Research \& Development.

PROTOCOL REGISTRATION: PROSPERO CRD42020187078

KEY WORDS: COVID-19 vaccine; health disparities; H1N1 vaccine; systematic review.

J Gen Intern Med 36(6):1734-45

DOI: $10.1007 / \mathrm{s} 11606-021-06715-7$

(C) This is a U.S. government work and not under copyright protection in the U.S.; foreign copyright protection may apply 2021

\section{BACKGROUND}

The distribution of COVID-19 vaccines is a public health campaign of unprecedented scope. Current estimates suggest that $60-90 \%$ of the US population will need to be vaccinated to reach herd immunity, depending on vaccine efficacy and duration of protection. ${ }^{1}$ Troubling levels of vaccine hesitancy, combined with longstanding disparities in vaccination rates (e.g., seasonal influenza), pose important challenges to COVID-19 vaccine uptake, particularly among vulnerable populations. For example, recent surveys suggest that compared to most groups, African American (AA)/Black participants are more hesitant about the COVID-19 vaccine. ${ }^{2,3}$

Compounding this problem is that disadvantaged populations also have higher rates of COVID-19 exposure and infection, ${ }^{4}$ bear a disproportionate burden of chronic illness that may make them more vulnerable to COVID- $19,{ }^{5}$ and often have limited access to healthcare. ${ }^{6}$ Strategic approaches are needed to understand and overcome barriers to vaccination among vulnerable populations.

The last time a new vaccine was developed for a global pandemic was during the 2009 influenza A virus subtype H1N1 "swine flu" epidemic. Vaccination patterns observed during the H1N1 pandemic may offer insight as the US ramps up its COVID-19 vaccination campaign. 
The aim of this systematic review was to understand disparities in H1N1 vaccine uptake by race/ethnicity, socioeconomic status (SES), rural/urban residence, population density, and disability status, and factors associated with unequal uptake, as well as the benefits and harms of interventions designed to attenuate inequities in $\mathrm{H} 1 \mathrm{~N} 1$ vaccine uptake - in an effort to address potential disparities in COVID-19 vaccine access and uptake.

\section{METHODS}

This study was part of a larger review commissioned by the Veterans Health Administration (VHA) focused on healthrelated inequalities in epidemics and pandemics pre-dating COVID-19. ${ }^{7}$ It includes the subset of studies from the parent review that focused on H1N1 vaccination. The protocol, which follows PRISMA guidelines, ${ }^{8}$ was registered to PROSPERO (CRD42020187078) before study initiation.

\section{Data Sources and Searches}

We searched MEDLINE ALL, PsycINFO, Cochrane Database of Systematic Reviews, and Cochrane Central Register of Controlled Trials from inception through May 8, 2020, using terms related to epidemics, pandemics, disasters, and health disparities, and a supplementary search of Ovid Medline targeting H1N1 vaccination on August 24, 2020. We reviewed the bibliographies of relevant articles and contacted experts to identify additional studies. Search strategies were developed in consultation with a research librarian (Appendix Item 1).

\section{Study Selection}

Eligible studies examined H1N1 vaccine-related inequalities by race/ethnicity, socioeconomic status (SES), disability, or urban/rural residence in US populations. Studies were independently screened for inclusion by two reviewers. Disagreements were resolved through consensus or a third reviewer (Appendix Table 1).

\section{Data Extraction and Quality Assessment}

We abstracted details related to sample size, setting, population characteristics, eligibility criteria, vaccination rates, and factors potentially contributing to vaccination inequalities. Data were abstracted by one investigator and confirmed by a second. Two reviewers independently assessed study risk-ofbias with the Newcastle-Ottawa Scale (NOS) for observational studies, ${ }^{9}$ and the Critical Appraisal Skills Programme (CASP) Qualitative Checklist for qualitative studies. ${ }^{10}$ Disagreements were resolved by consensus or a third reviewer.

\section{Data Synthesis and Analysis}

We qualitatively synthesized the evidence and present it in tables. Our approach was guided by the Social Ecological
Table 1 Social Ecological Framework for H1N1 Vaccine Uptake

\begin{tabular}{ll}
\hline \hline Category & Examples \\
\hline $\begin{array}{l}\text { Intrapersonal: attitudes and } \\
\text { beliefs }\end{array}$ & $\begin{array}{l}\text { Perceived risk from illness; perceived } \\
\text { safety of H1N1 vaccine; trust in } \\
\text { government's handling of the } \\
\text { pandemic; perceived presence in a } \\
\text { priority group; history of seasonal flu } \\
\text { vaccine acceptance; attitude toward }\end{array}$ \\
$\begin{array}{l}\text { H1N1 vaccine } \\
\text { Influence from friends, family, social }\end{array}$ \\
$\begin{array}{l}\text { networks; number of friends and } \\
\text { and social network norms }\end{array}$ & $\begin{array}{l}\text { vaccine; belief that friends and } \\
\text { family want them to be vaccinated }\end{array}$ \\
Institutional: healthcare & $\begin{array}{l}\text { Regular healthcare provider; Amount } \\
\text { of information from healthcare } \\
\text { provider about H1N1 and vacine }\end{array}$ \\
Commizations and providers & $\begin{array}{l}\text { Presence of disease; perceived risk in } \\
\text { the community; concern about } \\
\text { infecting others }\end{array}$ \\
dynamics & $\begin{array}{l}\text { Health insurance; priority group } \\
\text { status for vaccination }\end{array}$ \\
Policy: federal, state, local &
\end{tabular}

Social Ecological Model as described by Kumar et al. ${ }^{11}$

Model (SEM) - as utilized to describe H1N1 vaccine uptake in the USA (Table 1). ${ }^{11}$

We rated the strength of evidence (SOE) for H1N1 vaccine uptake by group using an established method that considers study limitations, directness, consistency, precision, and reporting bias to classify the SOE for each outcome independently as high, moderate, low, or insufficient, ${ }^{12}$ as well as separate guidance for applicability of the evidence. ${ }^{13}$

\section{RESULTS}

We reviewed 9098 titles and abstracts, and 163 full-text articles for the parent review, and identified 21 studies examining H1N1 vaccine uptake and its associated factors. We found no studies of interventions to mitigate $\mathrm{H} 1 \mathrm{~N} 1$ vaccine-related disparities (Fig. 1). Thirteen studies were nationally representative, cross-sectional surveys ${ }^{11,14-25}$; one was a national survey of Veterans with spinal cord conditions (two publications) ${ }^{26}$, ${ }^{27}$; four were regional or population-specific cross-sectional surveys $^{28-31}$; two were regional mixed-methods studies ${ }^{32,33}$; and one was a qualitative study performed in California. ${ }^{34}$ Table 2 contains study overviews; Appendix Table 2, detailed characteristics; Table 3, results; and Appendix Tables 3 and 4, ROB. Unless otherwise noted, comparators are White or higher SES populations.

\section{HIN 1 Vaccine Uptake}

We identified 11 studies (12 publications) reporting H1N1 vaccine receipt by group, ${ }^{11,14-16,18,19,26,27,30-33}$ and six

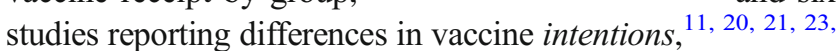
25,29 one of which examined willingness to receive the vaccine under a US Food and Drug Administration (FDA) emergency use authorization (EUA). ${ }^{23}$ Vaccination was selfreported in all but one study. ${ }^{33}$ Ten of 11 studies of vaccine 


\section{9,071 Citations identified from electronic database searches: \\ 7,090 from PubMed/Ovid MEDLINE May 1, 2020 \\ 1,946 from PsycINFO May 8, 2020 \\ 35 from Ovid EBM Reviews (CDSR, CCRCT) May 11, 2020}

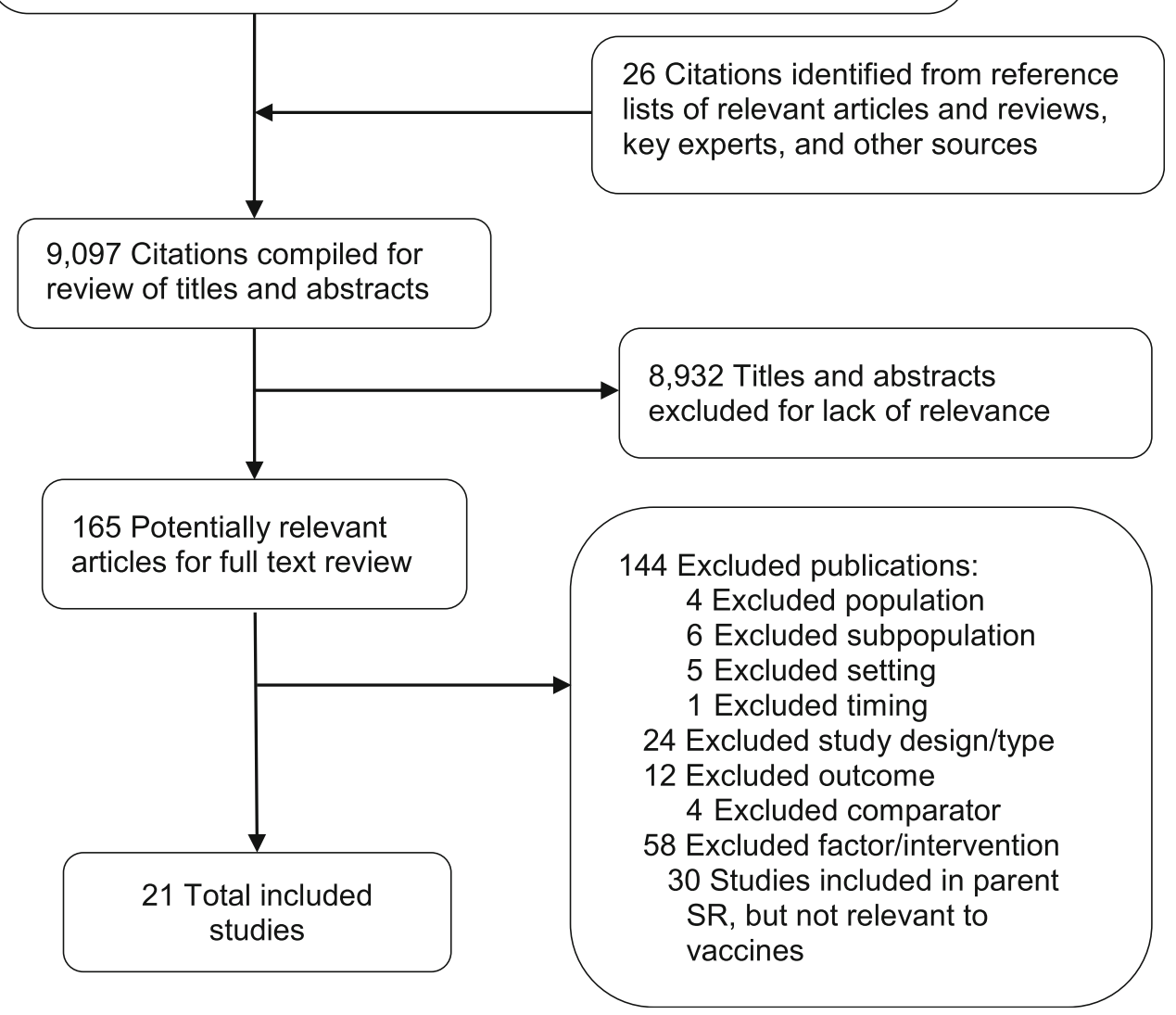

Figure 1 Literature flow diagram. Abbreviations: CCRCT, Cochrane Central Register of Controlled Trials; CDSR, Cochrane Database of Systematic Reviews; EBM, evidence-based Medicine; SR, systematic review.

receipt and five of six intention studies provided unadjusted data for at least one inter-group comparison. Studies reporting adjusted rates controlled for a wide range of factors. As such, SOE is based on unadjusted data (Table 4). We also report findings adjusted for sociodemographics where available. Differences by race/ethnicity were examined in 11 studies, by SES in nine, and by rural/urban residence in two. We identified no studies of uptake by disability status.

Vaccination Rates. By Racial/Ethnic Group. Seven studies provide moderate-strength evidence of lower H1N1 vaccination rates in AA/Blacks. ${ }^{11}$, 14-16, 27, 31, 33 Two national $(N=55,850 ; N=4040)^{1416}$ and one regional study $(N=1541)^{31}$ found that significantly fewer AA/Blacks reported receiving the $\mathrm{H} 1 \mathrm{~N} 1$ vaccine. In two other national studies $(N=2079 ; N=2355),{ }^{11,15}$ lower vaccine uptake was reported by AA/Blacks but the differences were not significant. A study of Veterans with spinal cord conditions reported no differences by race/ethnicity. ${ }^{27} \mathrm{~A}$ final study, by Plough et al. ${ }^{33}$ compared the racial and ethnic breakdown of the population vaccinated at free vaccination sites in Los Angeles (LA) County to estimates of each group's total county population. AA/Black residents were half as likely to receive the H1N1 vaccine despite targeted outreach. However, vaccinations at free sites accounted for only $20 \%$ of the vaccinations in LA County during the study period, leaving a high degree of uncertainty.

One additional study reported data adjusted for sociodemographic factors and found non-significantly lower vaccination rates among $\mathrm{AA} / \mathrm{Black}$ respondents. ${ }^{19}$

Eight studies provide low-strength evidence of lower H1N1 vaccination rates among Latinos. ${ }^{11,14-16,18,27,31,33}$ Two nationally representative studies $(N=55,850 ; N=11,834)$ and one in LA county ${ }^{31}$ found lower vaccination rates for Latinos. Three nationally representative studies $(N=2079 ; N=2355$; $N=4040)^{11,15,16}$ and one study of Veterans with spinal cord conditions $(N=3384)^{27}$ found no difference in vaccine uptake by Latino ethnicity; however, the directions of effects were largely consistent with lower rates. Plough et al.'s free vaccination site study in LA County found that Latinos were more likely to be vaccinated. ${ }^{33}$

One study found that foreign-born Latinos reported lower vaccination rates than those born in the USA. ${ }^{18}$ It also reported 
Table 2 Characteristics of Included Studies

\begin{tabular}{|c|c|c|c|c|}
\hline Author, year & $N$ & Study design & Location & Target population \\
\hline Boyd, $2013^{32}$ & 56 & $\begin{array}{l}\text { Mixed methods } \\
\text { (cross-sectional; qualitative) }\end{array}$ & Georgia state & Women enrolled in WIC (rural and urban) \\
\hline Burger, $2018^{18}$ & 11,834 & Cross-sectional survey & National & Representative (White and Latino) \\
\hline Cassady, $2012^{34}$ & 90 & Qualitative focus groups & California state & $\begin{array}{l}\text { Latino farmworkers, pregnant women, and indigenous } \\
\text { Mexicans with LEP }\end{array}$ \\
\hline $\begin{array}{l}\text { Etingen } 2012^{26} / \\
\text { LaVela }^{27}\end{array}$ & 3384 & Cross-sectional survey & National & Veterans with spinal cord-related disabilities \\
\hline Freimuth, $2014^{22}$ & 1543 & Cross-sectional survey & National & Representative \\
\hline Frew, $2012^{28}$ & 503 & Cross-sectional survey & Atlanta, GA & Racial and ethnic minorities \\
\hline Galarce, $2011^{19}$ & 1569 & Cross-sectional survey & National & Representative \\
\hline Gargano, $2011^{29}$ & 102 & Cross-sectional survey & 2 rural counties, GA & Secondary school staff \\
\hline Hernandez, $2019^{30}$ & 225 & Cross-sectional survey & $\begin{array}{l}2 \text { large, midwestern } \\
\text { cities }\end{array}$ & Pregnant women \\
\hline Kumar, 2012 11 & 2042 & Cross-sectional survey & National & Representative \\
\hline Lin, $2018^{25}$ & 1569 & Cross-sectional survey & National & Representative \\
\hline Mesch, $2014^{21}$ & 1000 & Cross-sectional survey & National & Representative \\
\hline Mesch, 2014 & 968 & Cross-sectional survey & National & Representative \\
\hline Plough, $2011^{33}$ & 163,087 & $\begin{array}{l}\text { Mixed methods (cross- } \\
\text { sectional; qualitative) }\end{array}$ & LA County, CA & $\begin{array}{l}\text { Free public vaccination sites (distributing } 20 \% \text { of LA } \\
\text { County's total vaccine supply) }\end{array}$ \\
\hline Quinn, $2009^{23}$ & 1543 & Cross-sectional survey & National & Representative \\
\hline Quinn, $2011^{24}$ & 1479 & Cross-sectional survey & National & Representative \\
\hline $\begin{array}{l}\text { Ramanadhan, } \\
2015^{17}\end{array}$ & 1166 & Cross-sectional survey & National & $\begin{array}{l}\text { Representative of those who had not received the H1N1 } \\
\text { vaccine }\end{array}$ \\
\hline Redelings, $2012^{31}$ & 1541 & Cross-sectional survey & LA County, CA & Public health clinic patients \\
\hline Santibanez, $2013^{14}$ & 55,850 & Cross-sectional survey & National & Representative \\
\hline SteelFisher, $2015^{15}$ & 2355 & Cross-sectional survey & National & Representative \\
\hline $\begin{array}{l}\text { Uscher-Pines, } \\
2011^{16}\end{array}$ & & Cross-sectional survey & National & Representative \\
\hline
\end{tabular}

Abbreviations: CA, California; GA, Georgia; LA, Los Angeles; LEP, limited English proficiency; WIC, Women, Infants and Children Program

adjusted data and found that controlling for demographics and comorbidities accounted for the disparity for US-born Latinos. Further adjustment for SES explained the disparity for foreignborn Latinos. $^{18}$

Three studies found similar or higher vaccination rates in Asian, American Indian/Alaska Native (AI/AN), and/or Pacific Islander populations. ${ }^{15,31,33}$ These studies, however, did not provide sufficient evidence to draw conclusions due to inconsistency in results across the studies, imprecise effect estimates, and limited generalizability.

By Socioeconomic Status. Four studies provide low-strength evidence that low-SES populations were less likely to be vaccinated. ${ }^{11}, 14,27,30$ One large nationally representative study $(N=55,850)^{14}$ and a small study of pregnant women in the Midwest $(N=225)^{30}$ found that significantly fewer lowSES adults reported receiving the H1N1 vaccine. One study found no difference in vaccine receipt by education or income ${ }^{11}$ and another found no difference by education. ${ }^{27}$

One study found that after controlling for age, gender, and race/ethnicity, those with a high school education reported lower vaccination rates than all other education levels. ${ }^{19}$

By Rurality. A small study ( $N=56$ ) found that fewer rural than urban participants reported being vaccinated. ${ }^{32}$ The evidence was insufficient to draw conclusions.

A nationally representative study $(N=1569)$ found that after controlling for age, gender, and SES, urban and rural residents reported similar rates of vaccination. ${ }^{19}$
Vaccine Intentions. By Racial/Ethnic Group. Three nationally representative studies $(N=2079 ; N=968 ; N=1000)^{10,19,20}$ and one regional study $(N=102)^{29}$ provide low-strength evidence that AA/Black participants were similar in reported intention to be vaccinated.

One study found that after adjusting for SES, age, and gender, AA/Black participants were more likely to intend to be vaccinated. ${ }^{11}$

The same three nationally representative studies provide low-strength evidence of Latinos' greater intention to be vaccinated. ${ }^{11,20,21}$ One found a significantly greater likelihood of intention to be vaccinated. ${ }^{11}$ The second found that more Latinos reported the intention to be vaccinated; however, the difference was not significant, ${ }^{21}$ and the third found no difference. $^{20}$

Two of the studies also reported adjusted results - one accounting for demographics ${ }^{11}$ and the second accounting for demographic factors plus $\mathrm{SES}^{21}$ —and found that Latinos remained more likely to intend to be vaccinated.

By Socioeconomic Status. The same studies provide lowstrength evidence of no difference in vaccination intentions by SES. ${ }^{11,20,21}$

Two studies controlled for demographic factors and found no difference in vaccine intentions by SES. ${ }^{21,25}$

Willingness to Receive H1N1 Vaccine Under EUA. A nationally representative study $(N=1543)$ examined willingness to accept a vaccine under FDA EUA. ${ }^{23}$ 


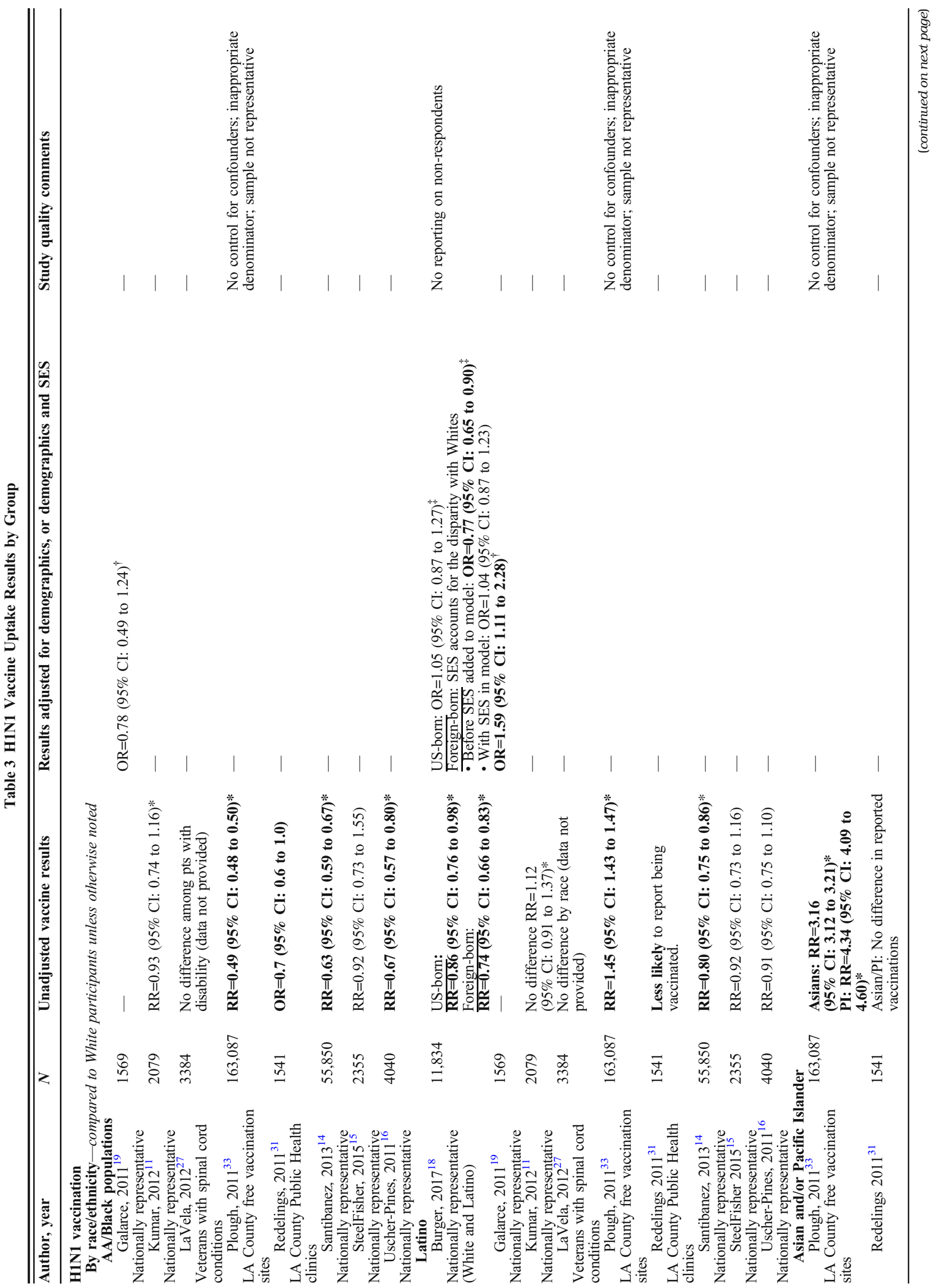




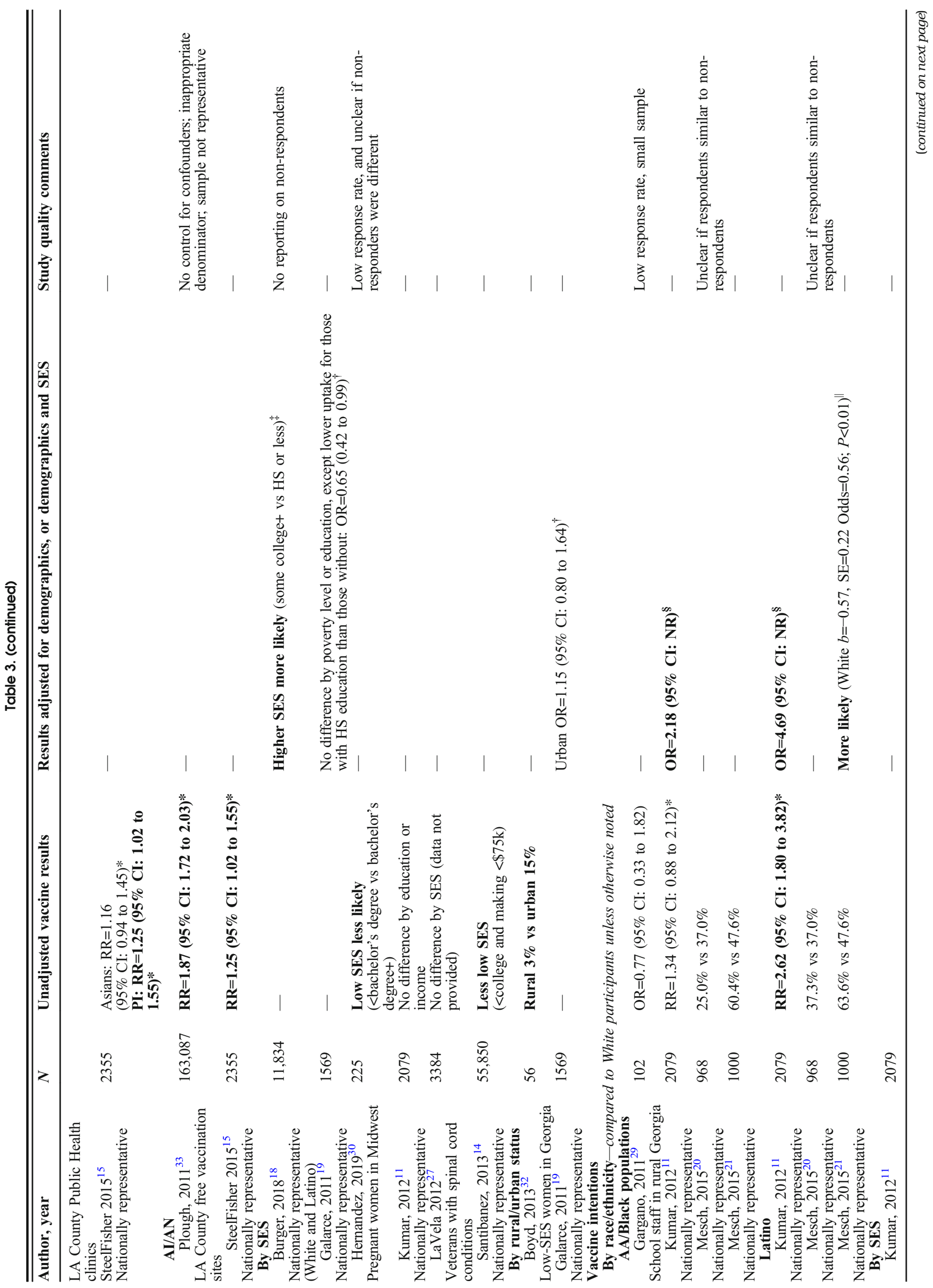




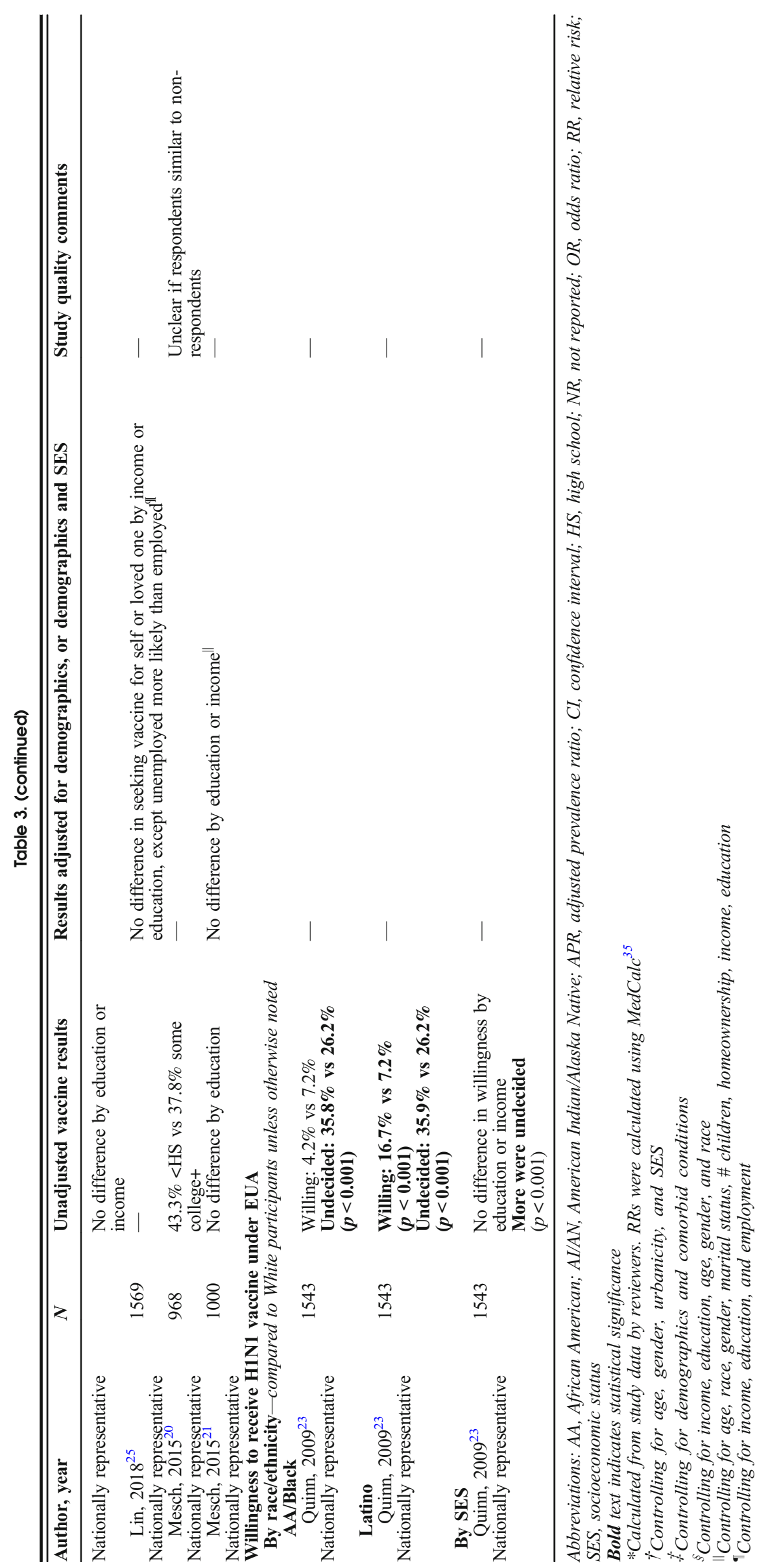


Table 4 Strength of the Evidence for Studies of H1N1 Vaccination or Vaccine Intentions

\begin{tabular}{|c|c|c|c|c|c|c|}
\hline $\begin{array}{l}\text { Population of } \\
\text { interest }\end{array}$ & $\begin{array}{l}\text { Comparator } \\
\text { population }\end{array}$ & $\begin{array}{l}\text { \# of } \\
\text { studies }\end{array}$ & $\begin{array}{l}\text { Vaccine } \\
\text { uptake } \\
\text { likelihood }\end{array}$ & $\begin{array}{l}\text { Strength of } \\
\text { evidence }\end{array}$ & SOE justification & Notes \\
\hline \multicolumn{7}{|l|}{ Vaccination } \\
\hline AA/Black & White & 7 & Less likely & Moderate & - & $\begin{array}{l}\text { Most studies (including the large, } \\
\text { good-quality studies), found signifi- } \\
\text { cantly lower uptake; all general } \\
\text { population studies found the same } \\
\text { direction of effect }\end{array}$ \\
\hline Latino & & 8 & Less likely & Low & Inconsistency & $\begin{array}{l}\text { Studies with non-significant differ- } \\
\text { ences largely found lower vaccination } \\
\text { rates; the } 2 \text { largest, good-quality } \\
\text { studies found that Latinos were sig- } \\
\text { nificantly less likely }\end{array}$ \\
\hline Asian & & 2 & Unclear & Insufficient & $\begin{array}{l}\text { Inconsistency, } \\
\text { imprecision, } \\
\text { indirectness }\end{array}$ & - \\
\hline $\mathrm{AI} / \mathrm{AN}$ & & 2 & Unclear & Insufficient & Indirectness & - \\
\hline Pacific Islander & & 1 & Unclear & Insufficient & Indirectness & - \\
\hline Asian/Pacific & & 1 & Unclear & Insufficient & Indirectness, & - \\
\hline $\begin{array}{l}\text { Islander } \\
\text { Lower SES } \\
\text { (education and/or }\end{array}$ & Higher SES & 4 & Less likely & Low & $\begin{array}{l}\text { imprecision } \\
\text { Inconsistency }\end{array}$ & - \\
\hline $\begin{array}{l}\text { income) } \\
\text { Rural }\end{array}$ & Urban & 1 & Unclear & Insufficient & $\begin{array}{l}\text { Single, small study } \\
\text { with multiple } \\
\text { limitations }\end{array}$ & - \\
\hline With disabilities & $\begin{array}{l}\text { Without } \\
\text { disabilities }\end{array}$ & 0 & No evidence & - & — & - \\
\hline \multicolumn{7}{|c|}{ Vaccine intentions } \\
\hline AA/Black & White & 4 & $\begin{array}{l}\text { No } \\
\text { difference }\end{array}$ & Low & Imprecision & - \\
\hline Latino & & 3 & More likely & Low & Inconsistency & - \\
\hline $\begin{array}{l}\text { Lower SES } \\
\text { (education and/or } \\
\text { income) }\end{array}$ & Higher SES & 3 & $\begin{array}{l}\text { No } \\
\text { difference }\end{array}$ & Low & Unclear precision & - \\
\hline Rural & Urban & 0 & No evidence & - & - & - \\
\hline With & Without & 0 & No evidence & - & - & - \\
\hline \multirow{2}{*}{\multicolumn{7}{|c|}{ Willingness to take H1N1 vaccine under FDA EUA }} \\
\hline & & & & & & \\
\hline AA/Black & White & 1 & Unclear & Insufficient & $\begin{array}{l}\text { Unclear } \\
\text { consistency-one } \\
\text { study }\end{array}$ & - \\
\hline Latino & & 1 & Unclear & Insufficient & $\begin{array}{l}\text { Unclear } \\
\text { consistency-one } \\
\text { study }\end{array}$ & - \\
\hline $\begin{array}{l}\text { Lower SES } \\
\text { (education and/or } \\
\text { income) }\end{array}$ & Higher SES & 1 & Unclear & Insufficient & $\begin{array}{l}\text { Unclear } \\
\text { consistency_one } \\
\text { study }\end{array}$ & - \\
\hline
\end{tabular}

Abbreviations: AA, African American; AI, American Indian; AN, Alaska Native; EUA, Emergency Use Authorization; FDA, Food \& Drug Administration; SES, socioeconomic status

Respondents were able to select "yes," "no," or "undecided." There was no difference in willingness among AA/Black respondents, though significantly more AA/Blacks were undecided. Latinos were more willing to accept the vaccine and also more likely to be undecided. Finally, there was no difference in willingness by SES, but a greater proportion of less educated participants were undecided. ${ }^{23}$

\section{Factors Associated with Vaccine Uptake}

Twenty-one studies examined factors associated with vaccination or vaccine intentions. Some of these studies examined whether disparities in $\mathrm{H} 1 \mathrm{~N} 1$ vaccine uptake were mediated by a given factor by analyzing whether the association between group status and vaccine uptake changed when that factor was incorporated in a statistical model. Other studies examined only whether group status was associated with the hypothesized mediator, without examining whether the mediator explained disparities in vaccine uptake. In qualitative studies, participants discussed factors potentially contributing to vaccine decisions. We have summarized key results by their corresponding construct of the SEM (detailed results in Appendix Table 5).

Intrapersonal. Perceptions of H1N1 Illness and Vaccine. Perceived susceptibility and illness severity. The largest national survey study found that more Latinos than Whites or AA/Blacks believed their chances of getting H1N1 were high if they were unvaccinated, as did those with the lowest education and income. ${ }^{14}$ Another national study found that those who were "very concerned" that someone in their family could get H1N1 were more likely to be vaccinated than those who were less concerned, and concern was higher for all minority groups. ${ }^{15}$ A small regional study found that among 
rural participants, fear of getting H1N1 was associated with increased vaccine intentions, but perceived severity of H1N1 was not. ${ }^{29}$ In two qualitative studies, perceptions of risk from H1N1 (severity and susceptibility) among vulnerable participants were generally low. ${ }^{32,34}$

Perceived effectiveness and safety of the vaccine. In the largest national survey study, belief in vaccine effectiveness was associated with uptake, and AA/Black and low-SES participants were less likely to believe the vaccine was effective in preventing H1N1. ${ }^{14}$ Multiple studies noted concerns over the novelty of the vaccine and whether it had been adequately tested. The same large study found more vaccine safety concerns among Latino participants, followed by AA/Blacks; however, fear of getting sick from the vaccine did not influence vaccine uptake. ${ }^{14}$ Another national survey found that belief in H1N1 vaccine safety was highly predictive of vaccination, and low-SES and urban participants were less likely to believe the vaccine was safe. ${ }^{19}$ In another, safety beliefs were associated with vaccination, and $\mathrm{AA} / \mathrm{Black}$ participants were less likely to believe the vaccine was safe. ${ }^{15}$ Two more studies, both in LA, found that $\mathrm{AA} / \mathrm{Black}$ participants were more concerned about safety and side effects of the H1N1 vaccine than other racial/ ethnic groups. ${ }^{31,33}$ One national study found no racial/ethnic differences in perceptions of vaccine safety, but in all groups the proportion who believed it was safe was less than $45 \% .{ }^{16}$ Fears about vaccine novelty and safety were also highlighted in qualitative studies. $^{32,34}$

Government and Provider Trust. A survey of racial and ethnic minority adults in Atlanta, GA, found that higher trust in the US government and greater disagreement with vaccine conspiracy beliefs were associated with intention to receive the H1N1 vaccine. ${ }^{28}$ In a national study, government trust was not associated with vaccine uptake for $\mathrm{AA} /$ Black or Latino participants, but a positive relationship existed for Whites. ${ }^{22}$ Another found higher trust in government for AA/Black and Latino participants. ${ }^{23}$ Qualitative studies noted vaccine hesitancy related to mistrust for some AA/Blacks and Latinos in the studied communities. ${ }^{33,34}$

Trust in healthcare providers was not examined for its association with $\mathrm{H} 1 \mathrm{~N} 1$ vaccine uptake, but one study in LA found that $\mathrm{AA} / \mathrm{Blacks}$ were less likely to report that they trusted providers when they recommend vaccines. ${ }^{31}$

Interpersonal. Influence from friends, family, and social networks was examined in three studies. ${ }^{28-30}$ A study of pregnant women in the Midwest found that while lower education predicted lower vaccination rates, the relationship was explained by a lower proportion of social network connections who were college-educated $\mathrm{H} 1 \mathrm{~N} 1$ vaccine supporters. ${ }^{30}$ One study examined normative approval (the belief that one's friends, family, and healthcare providers would approve of their being vaccinated) among minorities, and found no association with H1N1 vaccine uptake. ${ }^{28}$ Among rural residents, after controlling for other factors, talking with friends about H1N1 was not a significant predictor of vaccination. ${ }^{29}$

Institutional. H1N1 Information or Recommendation from Provider. AA/Black and Latino participants in a national survey reported receiving provider recommendations to be vaccinated at similar rates, but it was not clear whether recommendations led to uptake. ${ }^{15}$ The same study found that after controlling for sociodemographics, healthcare access, and attitude-related variables, AA/Black, Latino, and $\mathrm{AI} / \mathrm{AN}$ participants were more likely to have asked their healthcare provider about how to protect themselves from H1N1. ${ }^{15}$ Among Veterans with spinal cord conditions, those with adequate and accurate information about H1N1 were more likely to be vaccinated, and AA/Black, Latino, and less educated participants were more likely to report not receiving sufficient information. ${ }^{26}$ Finally, a qualitative study found that participants were motivated to be vaccinated by provider recommendations, but many hesitated to bring up vaccination themselves without provider prompting. ${ }^{32}$

Community. The study in Atlanta examined the relationship between community factors and intention to vaccinate. Among racial/ethnic minorities, perceived salience of H1N1 to one's community was associated with greater vaccine uptake. ${ }^{28}$ In a small regional study, rural residents were less concerned that the virus was widely circulating in their communities. $^{32}$

Policy. Health Insurance and Access. Four studies examined the association between health insurance coverage and H1N1 vaccine uptake. ${ }^{11,15,16,18}$ Health insurance coverage was associated with greater vaccine uptake in two studies, and minority and low-SES groups in those studies were less likely to have insurance coverage. ${ }^{15,}{ }^{18}$ In one of these studies, the health insurance status of foreign-born Latino respondents was a significant predictor of H1N1 vaccine receipt. ${ }^{18}$ Among all Latinos, those of lower SES were less likely to be vaccinated even after controlling for sociodemographics and insurance status. ${ }^{18}$ Another study found that AA/Black participants remained less likely to be vaccinated after controlling for sociodemographic factors and insurance status. ${ }^{16}$ However, a different national study did not find racial/ethnic differences in H1N1 vaccine uptake by insurance coverage, nor an association between insurance and vaccination. ${ }^{11}$

Priority Status. In a qualitative study, several pregnant women (an H1N1 vaccine priority group) were included, and many who were vaccinated had done so at the behest of their providers. However, those with concerns about vaccine safety refused. ${ }^{34}$ 
Vaccine Access. In one study, workplace vaccination was lower for Latinos, ${ }^{16}$ and in another, more AA/Black, Latino, and low-SES participants reported being unable to obtain a vaccine. ${ }^{19}$ Barriers identified in qualitative studies included work-related factors (e.g., inability to take time off due to inflexible scheduling, or potential loss of income or job), lack of vaccine availability, cost concerns, and inconvenient vaccination locations/settings. ${ }^{32,34}$

\section{Interventions to Reduce Disparities in $\mathrm{H} 1 \mathrm{~N} 1$ Vaccine Uptake}

We found no studies of interventions addressing H1N1 vaccine-related disparities.

\section{DISCUSSION}

We identified 21 studies examining inequalities in H1N1 vaccine uptake during the 2009-2010 flu season in the USA. Studies were largely cross-sectional; the majority were well conducted and adequately reported. The qualitative studies clearly reported methodology and findings. We identified no studies of interventions targeting disparities in $\mathrm{H} 1 \mathrm{~N} 1$ vaccine uptake. Low- to moderate-strength evidence indicated that vaccine uptake was lower in AA/Black, Latino, and lowSES populations. Evidence was insufficient to draw conclusions about disparities in vaccine uptake for Asian, $\mathrm{AI} / \mathrm{AN}$, and Pacific Islander populations or by rural/urban residency status, and there was no evidence for those with disabilities. Findings about intention to be vaccinated differed from those examining vaccine receipt. We found low-strength evidence that AA/Black populations were similar and that Latinos were more likely to intend to be vaccinated. There was no difference in intention by SES. There was no evidence for populations by rural/urban residency status or those with disabilities. Intention to be vaccinated under EUA did not differ by race/ethnicity or SES, but a greater share of these populations were undecided, albeit the evidence was insufficient.

These findings of similar or greater intentions to be vaccinated among minorities and low-SES groups suggest that lower H1N1 vaccination rates were related more to vaccine access than hesitancy. Studies in our review found that AA/ Black, Latino, and low-SES adults had lower rates of insurance coverage, and insurance coverage was associated with vaccine uptake. Insurance coverage may have provided financial access to vaccines, or it may even have facilitated uptake of free vaccines through increased healthcare connectedness. ${ }^{36}$ Other factors potentially affecting vaccine access included location and ability to take time off work. Indeed, exposurerelated factors likely help account for marked racial/ethnic COVID-19-related health disparities. ${ }^{4}$ Those who have the least work flexibility, such as frontline workers, also face the greatest exposure to COVID-19, highlighting the imperative to overcome these hurdles to vaccination.
Studies of H1N1 perceptions indicated that AA/Black, Latino, and low-SES populations generally had more concerns about contracting $\mathrm{H} 1 \mathrm{~N} 1$ and also more concerns about vaccine efficacy and safety. While these concerns did not translate into differences in intention to be vaccinated, there was some evidence that minorities and low-SES groups felt less adequately informed and were more likely to be undecided about a vaccine approved under EUA. Given that the vaccines for COVID-19 are also currently under EUA, this study is especially salient today and suggests that, although recent data indicate that many at-risk populations are unsure about the COVID-19 vaccine, ${ }^{37}$ efforts to help ease uncertainty are worthwhile and have the potential to increase equity.

The H1N1 pandemic happened over a decade ago, and COVID-19 is a very different pandemic. SARS-CoV-2 is more infectious and more widespread than H1N1 and has been much more widely disruptive to our daily lives, potentially limiting the applicability of our review to the present. The COVID-19 vaccines were developed rapidly (under an operation called "Warp Speed"), and some use novel mechanisms (e.g., mRNA). There are, however, many similarities - including the fact that both H1N1 and COVID are global pandemics that have disproportionately affected minority communities - that we believe allow lessons to be drawn from the H1N1 experience. In Text box 1, we suggest several recommendations, based on our review, that may help mitigate disparities in COVID-19 vaccine uptake.

Text box 1 Recommendations to improve equity of COVID-19 vaccinations

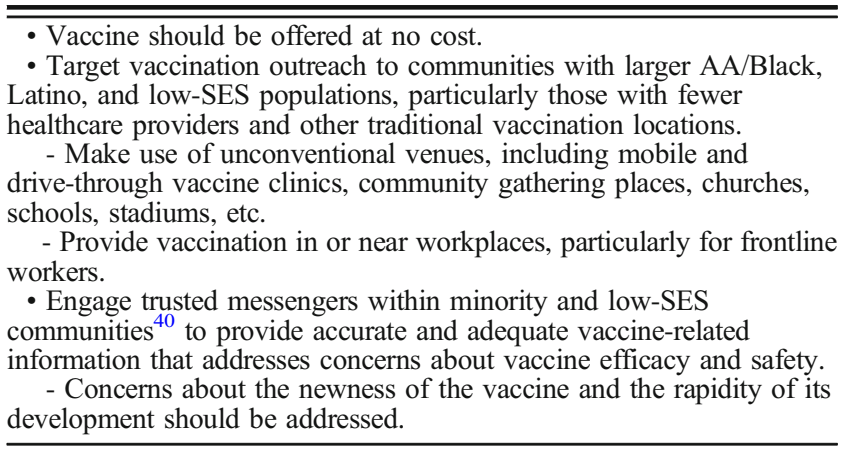

One salient factor that may have shifted since 2009-2010 is trust. Although trust in healthcare providers or government is an often-cited contributor to disparities in vaccine uptake, ${ }^{38}$ we did not find evidence that either played a strong role in H1N1 vaccine disparities. However, during the H1N1 pandemic, Barack Obama was president, and surveys indicated that minority groups had high levels of trust in President Obama and his administration. ${ }^{22}$ Since that time, misinformation about COVID-19 and vaccines on social media, ${ }^{39}$ racist and anti-immigrant federal policies, high-profile acts of police brutality in minority communities, and heightened awareness of structural racism and its contribution to disparities in COVID-19 infection and mortality, among myriad other factors, may have exacerbated minority and low-income 
communities' already fragile trust in government and healthcare institutions. ${ }^{40}$

There are also limitations of the evidence base. Studies were largely cross-sectional. Racial and ethnic groups were not consistently categorized across studies, and few or no studies examined disparities among Asian, Pacific Islander, and AI/ AN populations, or by rurality or disability status. The strength of evidence on H1N1 vaccine uptake was limited by imprecision, indirectness, and/or inconsistency, and vaccine uptake was measured by self-report in all but one study. No studies examined interventions or programs to mitigate vaccination disparities during the H1N1 pandemic. These are important areas for future disparities research.

Overall, our evidence review indicates that AA/Black, Latino, and low-SES adults were less likely to receive an H1N1 vaccine despite having similar or greater intent to receive it. Efforts to mitigate the identified causes of inequitable vaccine uptake from that pandemic will be essential to stem the disproportionate impact the COVID-19 pandemic has had on the same communities.

Supplementary Information The online version contains supplementary material available at https://doi.org/10.1007/s11606-02106715-7

Acknowledgements: The authors would like to acknowledge Robin Paynter, MLIS, for conducting the searches for this review.

Corresponding Author: Chelsea K. Ayers, MPH; VA Evidence Synthesis Program, VA Portland Health Care System, 3710 SW US Veterans Hospital Rd, Portland, OR 97239, USA (e-mail: chelsea. ayers@va.gov).

Funding This research was funded by the Department of Veterans Affairs, Veterans Health Administration, Health Services Research and Development.

\section{Declarations:}

Conflict of Interest: The authors declare that they do not have a conflict of interest.

Disclaimer: The findings and conclusions in this document are those of the author(s) who are responsible for its contents; the findings and conclusions do not necessarily represent the views of the Department of Veterans Affairs or the United States government. Therefore, no statement in this article should be construed as an official position of the Department of Veterans Affairs.

\section{REFERENCES}

1. Anderson RM, Vegvari C, Truscott J, Collyer BS. Challenges in creating herd immunity to SARS-CoV-2 infection by mass vaccination. The Lancet. 2020;396(10263):1614-6. doi: https://doi.org/10.1016/ S0140-6736(20)32318-7.

2. Malik AA, McFadden SM, Elharake J, Omer SB. Determinants of COVID-19 vaccine acceptance in the US. EClinicalMedicine. 2020;26:100495. doi: https://doi.org/10.1016/j.eclinm.2020.100495.

3. Kreps S, Prasad S, Brownstein JS, Hswen Y, Garibaldi BT, Zhang B, et al. Factors Associated With US Adults' Likelihood of Accepting COVID19 Vaccination. JAMA Network Open. 2020;3(10):e2025594-e. doi: https://doi.org/10.1001/jamanetworkopen.2020.25594.
4. Mackey K, Ayers CK, Kondo KK, Saha S, Advani SM, Young S, et al. Racial and Ethnic Disparities in COVID-19-Related Infections, Hospitalizations, and Deaths : A Systematic Review. Ann Intern Med. 2020. doi: https://doi.org/10.7326/m20-6306.

5. National Academies of Sciences, Engineering, and Medicine; Health and Medicine Division; Board on Population Health and Public Health Practice; Committee on Community-Based Solutions to Promote Health Equity in the United States; Baciu A, Negussie Y, Geller A, et al., editors. Communities in Action: Pathways to Health Equity. Washington (DC): National Academies Press (US); 2017 Jan 11. 2, The State of Health Disparities in the United States. Available from: https://www.ncbi.nlm. nih.gov/books/NBK425844/.

6. Agency for Healthcare Research and Quality. 2015 National Healthcare Quality and Disparities Report and 5th Anniversary Update on the National Quality Strategy: Access and Disparities in Access to Health Care Rockville, MD2015 [updated June 2018December, 21, 2020]. Available from: https://www.ahrq. gov/research/findings/nhqrdr/nhqdr15/access.html.

7. Kondo KK, Ayers CK, Williams BE, Kansagara D, Smith M, Mackey KM, Advani S, Saha S. Health Inequalities in Infectious Disease Epidemics Predating COVID-19 in the United States. Washington, DC: Evidence Synthesis Program, Health Services Research and Development Service, Office of Research and Development, Department of Veterans Affairs. 2020. VA ESP Project \#05225; Available from https://www.hsrd.research.va.gov/publications/esp/reports.cfm.

8. Moher D, Liberati A, Tetzlaff J, Altman DG. Preferred reporting items for systematic reviews and meta-analyses: the PRISMA statement. BMJ. 2009;339:b2535. doi: https://doi.org/10.1136/bmj.b2535.

9. Wells GA, Shea B, O'Connell D, Peterson J, Welch V, Losos M, et al. The Newcastle-Ottawa Scale (NOS) for assessing the quality of nonrandomised studies in meta-analyses. Available from: http://www. ohri.ca/programs/clinical_epidemiology/oxford.asp.

10. Critical Appraisal Skills Programme. CASP Qualitative Checklist 2018 [27 May 2020]. Available from: https://casp-uk.net/wp-content/uploads/ 2018/01/CASP-Qualitative-Checklist-2018.pdf.

11. Kumar S, Guinn SC, Kim KH, Musa D, Hilyard KM, Freimuth VS. The social ecological model as a framework for determinants of 2009 H1N1 influenza vaccine uptake in the United States. Health Education \& Behavior. 2012;39(2):229-43.

12. Berkman ND, Lohr KN, Ansari MT, Balk EM, Kane R, McDonagh M, et al. Grading the strength of a body of evidence when assessing health care interventions: an EPC update. J Clin Epidemiol. 2015;68(11):131224. doi: https://doi.org/10.1016/j.jclinepi.2014.11.023.

13. Atkins D, Chang SM, Gartlehner G, Buckley DI, Whitlock EP, Berliner E, et al. Assessing applicability when comparing medical interventions: AHRQ and the Effective Health Care Program. J Clin Epidemiol. 2011;64(11):1198-207. doi: https://doi.org/10.1016/j. jclinepi.2010.11.021.

14. Santibanez TA, Singleton JA, Santibanez SS, Wortley P, Bell BP. Socio-demographic differences in opinions about 2009 pandemic influenza A (H1N1) and seasonal influenza vaccination and disease among adults during the 2009-2010 influenza season. Influenza \& Other Respiratory Viruses. 2013;7(3):383-92.

15. SteelFisher GK, Blendon RJ, Kang M, Ward JR, Kahn EB, Maddox KE, et al. Adoption of preventive behaviors in response to the $2009 \mathrm{H} 1 \mathrm{~N} 1$ influenza pandemic: a multiethnic perspective. Influenza \& Other Respiratory Viruses. 2015;9(3):131-42.

16. Uscher-Pines L, Maurer J, Harris KM. Racial and ethnic disparities in uptake and location of vaccination for 2009-H1N1 and seasonal influenza. Am J Public Health. 2011;101(7): 1252-5.

17. Ramanadhan S, Galarce E, Xuan Z, Alexander-Molloy J, Viswanath $\mathbf{K}$. Addressing the Vaccine Hesitancy Continuum: An Audience Segmentation Analysis of American Adults Who Did Not Receive the 2009 H1N 1 Vaccine. Vaccines. 2015;3(3):556-78.

18. Burger AE, Reither EN, Hofmann ET, Mamelund SE. The Influence of Hispanic Ethnicity and Nativity Status on 2009 H1N1 Pandemic Vaccination Uptake in the United States. Journal of Immigrant \& Minority Health. 2018;20(3):561-8.

19. Galarce EM, Minsky S, Viswanath K. Socioeconomic status, demographics, beliefs and $\mathrm{A}(\mathrm{H} 1 \mathrm{~N} 1)$ vaccine uptake in the United States. Vaccine. 2011;29(32):5284-9.

20. Mesch GS, Schwirian KP. Social and political determinants of vaccine hesitancy: Lessons learned from the H1N1 pandemic of 2009-2010. American Journal of Infection Control. 2015;43(11):1161-5. 
21. Mesch GS, Schwirian KP. Confidence in government and vaccination willingness in the USA. Health Promotion International. 2015;30(2):213-21.

22. Freimuth VS, Musa D, Hilyard K, Guinn SC, Kim $\mathbf{K}$. Trust during the early stages of the $2009 \mathrm{H} 1 \mathrm{~N} 1$ pandemic. Journal of health communication. 2014;19(3):321-39. doi: https://doi.org/10.1080/10810730.2013 811323.

23. Quinn SC, Kumar S, Freimuth VS, Kidwell K, Musa D. Public willingness to take a vaccine or drug under Emergency Use Authorization during the 2009 H1N1 pandemic. Biosecur Bioterror. 2009;7(3):275-90. doi: https://doi.org/10.1089/bsp.2009.0041.

24. Guinn SC, Kumar S, Freimuth VS, Musa D, Casteneda-Angarita N, Kidwell K. Racial disparities in exposure, susceptibility, and access to health care in the US H1N1 influenza pandemic. Am J Public Health 2011;101(2):285-93.

25. Lin L, McCloud RF, Jung M, Viswanath K. Facing a Health Threat in a Complex Information Environment: A National Representative Survey Examining American Adults' Behavioral Responses to the 2009/2010 A(H1N1) Pandemic. Health Educ Behav. 2018;45(1):77-89. doi: https:// doi.org/10.1177/1090198117708011.

26. Etingen B, LaVela SL, Miskevics S, Goldstein B. Health information during the H1N1 influenza pandemic: Did the amount received influence infection prevention behaviors? Journal of Community Health: The Publication for Health Promotion and Disease Prevention 2013;38(3):443-50. doi: https://doi.org/10.1007/s10900-012-9647-8.

27. Lavela SL, Goldstein B, Etingen B, Miskevics S, Weaver FM. Factors Associated With H1N1 Influenza Vaccine Receipt in a High-Risk Population During the 2009-2010 H1N1 Influenza Pandemic. Topics in Spinal Cord Injury Rehabilitation. 2012;18(4):306-14.

28. Frew PM, Painter JE, Hixson B, Kulb C, Moore K, del Rio C, et al. Factors mediating seasonal and influenza A (H1N1) vaccine acceptance among ethnically diverse populations in the urban south. Vaccine. 2012;30(28):4200-8.

29. Gargano LM, Painter JE, Sales JM, Morfaw C, Jones LM, Weiss P, et al. Correlates of 2009 pandemic H1N1 influenza vaccine acceptance among middle and high school teachers in rural Georgia. Journal of School Health. 2011;81(6):297-303.

30. Hernandez EM, Pullen E, Brauer J. Social Networks and the Emergence of Health Inequalities Following a Medical Advance: Examining Prenatal H1N1 Vaccination Decisions. Social Networks. 2019;58:156-67.

31. Redelings MD, Piron J, Smith LV, Chan A, Heinzerling J, Sanchez KM, et al. Knowledge, attitudes, and beliefs about seasonal influenza and H1N1 vaccinations in a low-income, public health clinic population. Vaccine. 2012;30(2):454-8.
32. Boyd CA, Gazmararian JA, Thompson ww. Knowledge, attitudes, and behaviors of low-income women considered high priority for receiving the novel influenza A (H1N1) vaccine. Maternal \& Child Health Journal. 2013;17(5):852-61.

33. Plough A, Bristow B, Fielding J, Caldwell S, Khan S. Pandemics and health equity: lessons learned from the H1N1 response in Los Angeles County. Journal of Public Health Management \& Practice. 2011;17(1):20-

34. Cassady D, Castaneda X, Ruelas MR, Vostrejs MM, Andrews T, Osorio L. Pandemics and vaccines: perceptions, reactions, and lessons learned from hard-to-reach Latinos and the H1N1 campaign. Journal of Health Care for the Poor \& Underserved. 2012;23(3):1106-22.

35. MedCalc. Free statistical calculators: Relative risk calculator [2 October 2020]. Available from: https://www.medcalc.org/calc/relative_risk.php.

36. Budhwani H, De P. Disparities in influenza vaccination across the United States: Variability by minority group, Asian sub-populations, socioeconomic status, and health insurance coverage. Public Health 2016; 138: 146-53. doi: https://doi.org/10.1016/j.puhe.2016.04.003.

37. Hamel L, Kirzinger A, Lopes L, Kearney A, Sparks G, Brodie M. KFF COVID-19 Vaccine Monitor: January 2021: Kaiser Family Foundation,; 2021 [updated January 22February 2, 2021]. Available from: https:// www.kff.org/report-section/kff-covid-19-vaccine-monitor-january-2021vaccine-hesitancy/.

38. Laine C, Cotton D, Moyer DV. COVID-19 Vaccine: Promoting Vaccine Acceptance. Ann Intern Med. 2020. doi: https://doi.org/10.7326/m208008.

39. Abbasi J. COVID-19 Conspiracies and Beyond: How Physicians Can Deal With Patients' Misinformation. JAMA. 2021;325(3):208-10. doi: https:// doi.org/10.1001/jama.2020.22018.

40. Alsan M, Stanford FC, Banerjee A, Breza E, Chandrasekhar AG Eichmeyer S, et al. Comparison of Knowledge and Information-Seeking Behavior After General COVID-19 Public Health Messages and Messages Tailored for Black and Latinx Communities : A Randomized Controlled Trial. Ann Intern Med. 2020.. doi: https://doi.org/10.7326/m20-6141.

Publisher's Note: Springer Nature remains neutral with regard to jurisdictional claims in published maps and institutional affiliations. 